\title{
Acute intussusception and polyp with malignant transformation in Peutz-Jeghers syndrome: A case report
}

\author{
JUAN YU and WEI JIANG \\ Department of General Surgery, Tianjin First Central Hospital, Tianjin 300192, P.R. China
}

Received July 20, 2014; Accepted May 7, 2015

DOI: $10.3892 / 01.2015 .3330$

\begin{abstract}
Intussusception is one of the most frequent complications of Peutz-Jeghers syndrome, and has been well described in previous studies. More attention has been paid to malignancy, which is another complication of Peutz-Jeghers syndrome and which leads to increased mortality. Few cases of intussusception combined with malignant polyps in Peutz-Jeghers syndrome have been reported to date. In the present study, we report a case of intussusception and malignant polyps occurring in various parts of the small intestine in a 43 -year-old male. In addition to repair of the intussusception and partial resection of the small intestine with malignant polyps, we also simultaneously performed polypectomy of as many polyps as possible without resection of the small intestine. Our aim is to make clinicians aware of intussusception and malignant polyps coexisting in Peutz-Jeghers syndrome when performing emergency surgery. Prophylaxis and polypectomy of the entire small bowel is an effective way to reduce the frequency of laparotomies in patients with this disease.
\end{abstract}

\section{Introduction}

Peutz-Jeghers syndrome is a rare autosomal dominant inherited disease characterized by mucocutaneous pigmentations and gastrointestinal polyps (1). It was first described by Peutz (2) in 1921 and Jeghers et al (3) in 1949. The incidence has been estimated to be between 1 in 8,300 and 1 in 200,000 live births (4). The polyps are located predominantly in the small intestine, and may cause various complications, including abdominal pain, anemia and acute intussusceptions (5). Patients with Peutz-Jeghers syndrome have an increased risk of developing cancer or transformation to malignant polyps in the gastroinstestinal tract and

Correspondence to: Miss. Juan Yu, Department of General Surgery, Tianjin First Central Hospital, 24 Fukang Road, Nankai, Tianjin 300192, P.R. China

E-mail: yj8313@163.com

Key words: intussusception, Peutz-Jeghers syndrome, malignant polyp other organs (6). The coexistence of adult intussusception caused by Peutz-Jeghers syndrome and malignant polyps is rarely reported. Here, we report a case of intussusception and malignant polyps occurring in the small intestine in a 43-year-old male. The patient provided written informed consent to the publication of this case.

\section{Case report}

Patient presentation. A previously healthy 43 -year-old male presented to the emergency room at Tianjin First Central Hospital, China, with a 3-day history of nausea, vomiting and increasing crampy abdominal pain. The patient had previously undergone resection of the small intestine for intussusception. He had no recent weight loss and no family history of bowel disease.

Upon examination, the patient had a tender distended abdomen with no peritoneal signs, fever or adenopathy. The patient's skin, including the perioral area, appeared normal, as did the oral mucosa. Clear evidence of intestinal peristalsis in the left upper abdomen was observed when the patient experienced abdominal pain, and an increase in bowel sounds was noted. Laboratory tests indicated normal hemoglobin levels, blood count and liver and renal function. An erect abdominal radiograph of the patient revealed air-fluid levels. Subsequent computed tomography (CT) imaging of the abdomen revealed features typical of intussusception (Fig. 1).

Surgery and diagnosis. At surgery, part of the proximal jejunum, $20 \mathrm{~cm}$ away from the Treitz ligament, was observed to be invaginated into the proximal jejunum, which determined the diagnosis of intussusception (Fig. 2A). A mass of $\sim 3 \mathrm{~cm}$ could be felt at the lead point of the intussusception. Following the removal of the intussusception, this polyp was completely resected (Fig. 2B). There were numerous larger and smaller polyps identified by palpation and intermittent opening of the bowel which were also completely resected (Fig. 2C). The malignancy in the small intestine, $15 \mathrm{~cm}$ away from the ileocecal valve, involved the intestinal wall and appeared white and cauliflower-like. Partial resection of the small intestine with ileo-ileo anastomosis was performed (Fig. 3A). Through histopathological examination carried out following surgery, the mass was identified as a solitary Peutz-Jeghers polyp with malignant features (Fig. 3B). 


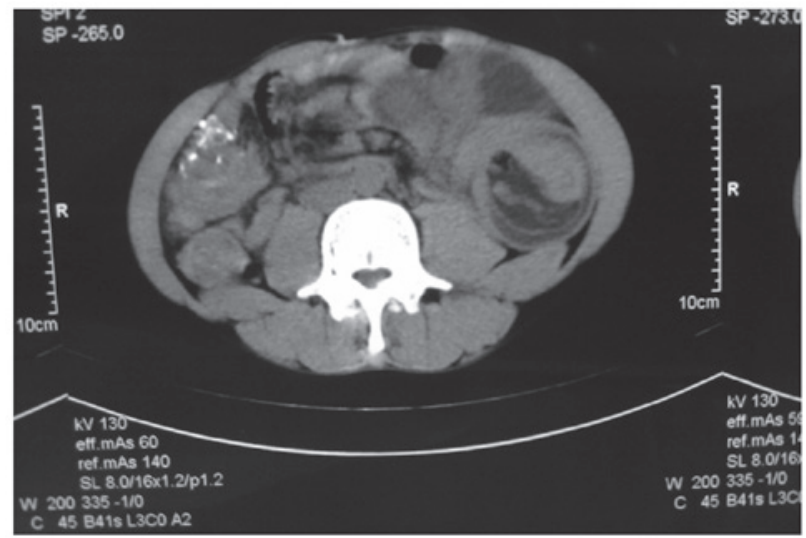

Figure 1. Computed tomography scan revealing the intussusception.
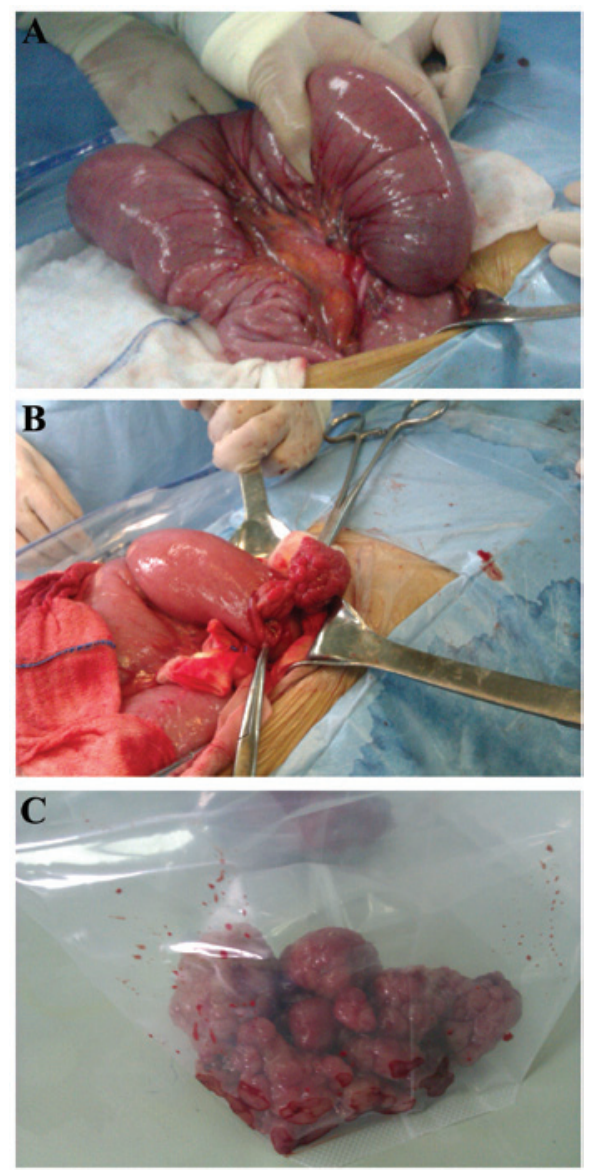

Figure 2. Surgical images. (A) Proximal jejunum intussusception. (B) Polyp that acted as a lead point for the intussusception. (C) Larger and smaller completely resected polyps.

Follow-up. The patient recovered with no further events and was discharged 10 days after surgery. He remained well 20 months later, and follow-up is ongoing.

\section{Discussion}

Peutz-Jeghers syndrome is a rare, autosomal dominant inherited disorder, which is characterized by mucocutaneous pigmentations, gastrointestinal polyposis and an increased risk of cancer (1). The sites most commonly affected
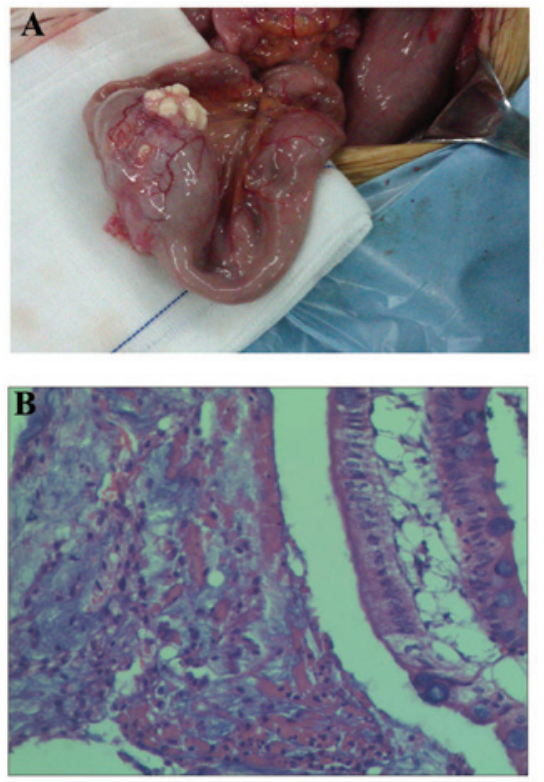

Figure 3. (A) The malignancy invaded the intestinal wall and was white and cauliflower-like. (B) Histopathological examination revealed polyps with malignant transformation invading the deep muscular layer outside the serosa in the small intestine (hematoxylin and eosin staining; original magnification, $\mathrm{x} 200$ ).

by Peutz-Jeghers polyps in the gastrointestinal tract are the jejunum, colorectal region, duodenum and stomach, in decreasing order of frequency. The most common histological type of polyps is hamartoma, which exhibits epithelial elements in the submucosa, muscularis propria and subserosa. The polyps often surround mucin-filled spaces. These hamartomas may induce significant complications including intussusception or gastrointestinal bleeding, requiring a number of laparotomies and bowel resections. During surgery for intestinal intussusception, it is preferable to simultaneously resect as many polyps as possible to prevent recurrence of the complications they cause (7).

Peutz-Jeghers syndrome patients are at a higher risk of cancer, and hence have an increased mortality rate. The incidence of tumors in patients with Peutz-Jeghers syndrome patients is 15 times higher than that observed in normal individuals, and the incidence of malignant tumors may be as high as $20 \%$ (8). Malignancy in Peutz-Jeghers syndrome is considered to arise in adulthood, and occurs only rarely in children (9). In addition to the hamartomas, malignant tumors of other organs outside the gastrointestinal tract also evolve into adenomas and carcinomas in a process occurring via de novo pathways (10). STK11/LKB1 (hereafter referred to as STK11) germline mutations account for the majority of cases of Peutz-Jeghers syndrome, whereas large deletions account for $\sim 30 \%$ of cases (11). In addition, malignant polyps may be associated with interferon-induced transmembrane glycoprotein-1 (12), which may be an effective marker to test for polyps in the process of malignant transformation in Peutz-Jeghers syndrome. The main therapeutic treatment used for Peutz-Jeghers syndrome is non-steroidal anti-inflammatory drugs. Wei et al (13) also demonstrated that rapamycin inhibits the enzyme encoded by STK11, which inhibited the growth of hamartoma. 
Indications for surgery include polyps with abdominal cramps, intestinal bleeding, intussusception, intestinal obstruction and malignant polyps confirmed by endoscopic biopsy. As a result of the risk of complications related to the hamartomatous polyps and the increased risk of cancer, prophylaxis and polypectomy of the entire small bowel are effective ways of reducing the frequency of laparotomies in patients with Peutz-Jeghers syndrome (14). The combination of surgical and endoscopic polypectomy is an efficient and practical choice in the management of polyps in patients with the disease (7). Radical surgery is required only if the polyps are confirmed to be malignant.

\section{References}

1. Korsse SE, van Leerdam ME and Dekker E: Peutz-Jeghers syndrome. Ned Tijdschr Tandheelkd 120: 12-16, 2013 (In Dutch).

2. Peutz J: A very remarkable case of familial polyposis of mucous membrane of intestinal tract and accompanied by peculiar pigmentations of skin and mucous membrane. Nederl Maandschr Geneesk 10: 134-146, 1921.

3. Jeghers H, McKusick VA and Katz KH: Generalized intestinal polyposis and melanin spots of the oral mucosa, lips and digits; a syndrome of diagnostic significance. N Engl J Med 241: 1031-1036, 1949

4. Cienfuegos JA, Baixauli J, Zozaya G, Bueno A, Arredondo J, Regueira FM, Angós R, Hernández-Lizoáin JL and Idoate MA: Peutz-Jeghers syndrome and duodeno-jejunal adenocarcinoma - therapeutic implications. Rev Esp Enferm Dig 101: 875-879, 2009

5. Wang H, Luo T, Liu WQ, Huang Y, Wu XT and Wang XJ: Clinical presentations and surgical approach of acute intussusception caused by Peutz-Jeghers syndrome in adults. J Gastrointest Surg 15: 2218-2225, 2011.
6. Riegert-Johnson D, Roberts M, Gleeson FC, Krishna M and Boardman L: Case studies in the diagnosis and management of Peutz-Jeghers syndrome. Fam Cancer 10: 463-468, 2011.

7. Miyachi T, Tanaka N, Endo K, Fujishima F, Sasaki H, Nagao M, Morikawa T, Naitoh T, Miura K, Shibata C, et al: A case of Peutz-Jeghers syndrome with repeated small intestinal intussusception successfully treated by intraoperative endoscopic polypectomy. Nihon Shokakibyo Gakkai Zasshi 110: 1014-1021, 2013 (In Japanese).

8. van Lier MG, Westerman AM, Wagner A, Looman CW, Wilson JH, de Rooij FW, Lemmens VE, Kuipers EJ, Mathus-Vliegen EM and van Leerdam ME: High cancer risk and increased mortality in patients with Peutz-Jeghers syndrome. Gut 60: 141-147, 2011.

9. Wangler MF, Chavan R, Hicks MJ, Nuchtern JG, Hegde M, Plon SE and Thompson PA: Unusually early presentation of small-bowel adenocarcinoma in a patient with Peutz-Jeghers syndrome. J Pediatr Hematol Oncol 35: 323-328, 2013.

10. Taguchi T, Suita S, Taguchi S and Tanaka S: Peutz-Jeghers syndrome in children: high recurrence rate in short-term follow-up. Asian J Surg 26: 221-224, 2003.

11. Resta N, Giorda R, Bagnulo R, Beri S, Della Mina E, Stella A, Piglionica M, Susca FC, Guanti G, Zuffardi O and Ciccone R: Breakpoint determination of 15 large deletions in Peutz-Jeghers subjects. Hum Genet 128: 373-382, 2010.

12. Ma Y, Zhang G, Fu X, Xia O, Zhan C, Li L, Wang Z and Wu B: Wnt signaling may be activated in a subset of Peutz-Jeghers syndrome polyps closely correlating to LKB1 expression. Oncol Rep 23: 1569-1576, 2010.

13. Wei C, Amos CI, Zhang N, Wang X, Rashid A, Walker CL, Behringer RR and Frazier ML: Suppression of Peutz-Jeghers polyposis by targeting mammalian target of rapamycin signaling. Clin Cancer Res 14: 1167-1171, 2008.

14. Wang H, Luo T, Liu WQ, Huang Y, Wu XT and Wang XJ: Clinical presentations and surgical approach of acute intussusception caused by Peutz-Jeghers syndrome in adults. J Gastrointest Surg 15: 2218-2225, 2011 